\title{
The structure and emission of an inner disk and a corona in the low/hard state
}

\section{Erlin Qiao and Bifang Liu}

\author{
National Astronomical Observatories, Chinese Academy of Sciences, \\ Beijing 100012, China \\ email: qiaoel@nao.cas.cn
}

\begin{abstract}
Recent observations reveal that a cool disk may survive in the innermost stable circular orbit (ISCO) for some black hole X-ray binaries in the low/hard state. The spectrum is characterized by a power law with a photon index $\Gamma \sim 1.5-2.1$ in the range of $2-10 \mathrm{keV}$ and a weak disk component with temperature of $\sim 0.2 \mathrm{keV}$. The formation of such a cool disk in the most inner region of black hole X-ray binaries at the low/hard state is investigated within the framework of disk accretion fed by condensation of hot corona. We also calculate the emergent spectra of the inner disk and corona. It's found that our model can very well explain the spectral features of GX 339-4 and Cyg X-1, in which the thin disk may exist at ISCO in the low/hard state.
\end{abstract}

\section{Introduction}

The picture of truncated outer thin disk + inner ADAF model in the low/hard state of black hole X-ray binaries (BHXRBs) is challenged by the recent observations Reis et al. (2010), in which the hard X-ray continuum is characterized by a power law with a photon index in the range of $\Gamma \sim 1.5-2.1$, a thermal component with a color temperature consistent with $L \propto T^{4}$ is detected in all eight sources. Both the $L \propto T^{4}$ relation and the broad iron line profile suggest that a cool disk extends to ISCO in the low/hard state.

A disk accretion model maintained by the recondensation of hot corona has been proposed to understand the presence of the cool disk in the most inner region around the black hole in the low/hard state by Liu et al. (2007). We calculate the structure of the corona and the emergent spectra of the inner disk-corona system Qiao \& Liu (2012). We briefly introduce the condensation model and show some numerical results in section 2. Section 3 is the conclusion.

\section{The condensation model and results}

It'found that when the accretion rate decreases just below the transition rate between high/soft state and low/hard state, as a consequence of efficient evaporation, the thin disk truncates at a distance where the maximum evaporation rate occurs (Liu et al.2002). The remnant disk from the truncation radius inwards can exist steadily fed by the condensation of the hot corona/ADAF rather than be swallowed by the black hole within the viscous time scale (Liu et al. 2007; Taam et al. 2008). The size of the inner remnant disk is governed by the accretion rate.

Given a black holes mass $m$, the accretion rate $\dot{m}$, the viscosity parameter $\alpha$ and the albedo $a$, the structure of the corona in radial direction is solved self-consistently. For $m=10, \alpha=0.2$, and $a=0.15$, we plot the accretion rate in the accretion disk and the accretion rate in the corona as functions of radius in the left panel of Fig. 1. It can be 

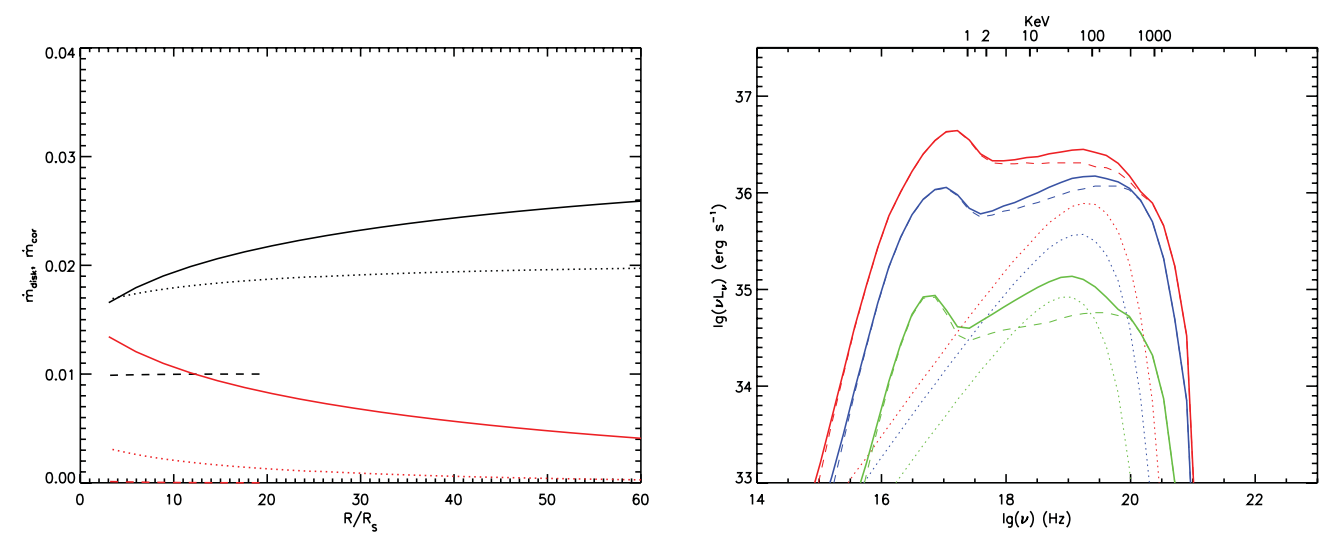

Figure 1. Left panel:The mass accretion rate in the accretion disk and the mass accretion rate in the corona as functions of radius. The red line and black line represent the mass accretion rate in the disk and the mass accretion rate in the corona respectively. The solid line, dotted line and dashed line are for $\dot{m}=0.03, \dot{m}=0.02$ and $\dot{m}=0.01$ respectively. Right panel: The emergent spectra of inner disk and corona. The red line, blue line and green line are the spectra for $\dot{m}=0.03, \dot{m}=0.02$ and $\dot{m}=0.01$ respectively. The solid line is the total spectrum, the dashed line is the contribution from the Comptonization of the soft photons of the thin disk by the electrons in the hot corona, the dotted line is the contribution of the bremsstrahlung from the transition layer. In all the calculations, $\alpha=0.2$ and albedo $a=0.15$ are adopted.

seen that, with increase of the accretion rate, more gases in the corona recondense back to the disk, the accretion rate in the disk increases.

The emergent spectra of inner disk and corona with mass accretion rate are plotted in the right panel of Fig. 1. The X-ray spectrum in the range of $2-10 \mathrm{keV}$ is roughly characterized by a power law with a photon index $\Gamma=1.63$ at $\dot{m}=0.01$ (solid green line). With increase of the mass accretion rate, the hard X-ray photon index is $\Gamma=1.75$ at $\dot{m}=0.02$ (solid blue line) and $\Gamma=1.92$ at $\dot{m}=0.03$ (solid red line). It's obviously that the X-ray spectra become softer with increase of the mass accretion rate. This can be understood as, the hard X-rays are dominated by Bremsstrahlung at low accretion rate, whereas by Compton radiation at high accretion rate.

Our model can successfully explain the spectral features of GX 339-4 and Cyg X-1, in which the thin disk may exist at ISCO in the low/hard state. For the detailed fitting results, one can see (Qiao \& Liu 2012).

\section{Conclusion}

We study the formation of a cool, optically thick, geometrically thin disk in the most inner region of black hole X-ray binaries in the low/hard spectral state within the context of disk accretion fed by condensation of hot corona and calculate the emergent spectra. Our model can very well explain the spectral features of GX 339-4 and Cyg X-1 in the low/hard spectral state, in which the thin disk may reside in ISCO.

\section{References}

Liu, B. F., Mineshige, S., Meyer, F. et al., 2002, ApJ, 575, 117

Liu, B. F., Taam, R. E., Meyer, F., \& Meyer-Hofmeister, E. 2007, ApJ, 671, 695

Qiao, Erlin, Liu, B. F. 2012, ApJ, 744, 145

Reis, R. C., Fabian, A. C., \& Miller, J. M., 2010, MNRAS, 402, 836

Taam, R. E., Liu, B. F., Meyer, F., \& Meyer-Hofmeister, E. 2008, ApJ, 688, 527 